Check for updates

Cite this: Soft Matter, 2020,

16,9183

Received 19th August 2020,

Accepted 24th September 2020

DOI: 10.1039/d0sm01512a

rsc.li/soft-matter-journal

\section{Light-responsive self-assembly of a cationic azobenzene surfactant at high concentration $\dagger$}

\author{
Camille Blayo, ${ }^{a}$ Elaine A. Kelly, (D) ${ }^{b}$ Judith E. Houston, (D) ${ }^{c}$ Nikul Khunti, ${ }^{d}$ \\ Nathan P. Cowieson ${ }^{d}$ and Rachel C. Evans (D)*b
}

The formation of high-concentration mesophases by a cationic azobenzene photosurfactant is described for the first time. Using a combination of polarised optical microscopy and small-angle X-ray scattering, optically anisotropic, self-assembled structures with long-range order are reported. The mesophases are disrupted or lost upon UV irradiation.

Dynamic structures based on non-covalent interactions offer significant benefits such as reversibility and responsiveness to stimuli. ${ }^{1-3}$ Of particular interest are simple, single-component systems with well-defined dimensions and morphologies and a rapid response to the stimulus. Specifically, light is a desirable stimulus due to its high degree of spatiotemporal resolution. Applications of light-responsive structures based on non-covalent interactions include controlled uptake and release, ${ }^{4,5}$ gelation, ${ }^{6-8}$ drug delivery ${ }^{1,9}$ and catalysis. ${ }^{10}$

The azobenzene unit has been widely used to create lightresponsive assemblies and nanoarchitectures,${ }^{11,12}$ due to its reversible photoisomerisation between cis- and trans-isomers upon UV and blue light irradiation, respectively. ${ }^{13,14}$ The more thermodynamically stable trans-isomer is linear, planar and apolar, while the pseudo-stable cis-isomer is bent, non-planar and has a dipole moment of $3 \mathrm{D}^{15,16}$ The significant change in polarity and geometry upon photoisomerisation has led to azobenzene being incorporated into surfactant structures, as these factors govern the key behaviours of surfactants i.e. surface activity and self-assembly. The resulting azobenzene photosurfactants (AzoPS), especially those with a trimethylammonium bromide

\footnotetext{
${ }^{a}$ School of Chemistry, Trinity College Dublin, College Green, Dublin 2, Ireland

${ }^{b}$ Department of Materials Science and Metallurgy, 27 Charles Babbage Road, CB3 OFS Cambridge, UK. E-mail: rce26@cam.ac.uk

${ }^{c}$ European Spallation Source (ESS), Odarslövsvägen 113, 22592 Lund, Sweden

${ }^{d}$ Diamond Light Source, Harwell Science and Innovation Campus, Didcot, Oxfordshire, OX11 ODE, UK

$\dagger$ Electronic supplementary information (ESI) available: Determination of the photostationary state, photoisomerisation kinetics, additional POM images, temperature-dependent SAXS measurements, DSC data. See DOI: 10.1039/ dosm01512a
}

head group (AzoTABs), have found success in areas such as DNA compaction, ${ }^{17-19}$ photo-controlled protein folding, ${ }^{20,21}$ droplet manipulation $^{22,23}$ and on-demand foam stability. ${ }^{24,25}$ In tandem with these advances, there have been several studies on the selfassembly behaviour of AzoTABs at low concentration, ${ }^{4 a, b, 26,27}$ but as yet no reports of their high-concentration behaviour. While the formation of lyotropic liquid crystal (LLC) phases has been described for neutral AzoPS, ${ }^{28-31}$ no such accounts exist for AzoTABs. AzoTABs generally have fewer hydrogen bonding sites than their neutral counterparts, which is expected to hinder their LLC phase-forming ability. ${ }^{32}$ The formation of lightresponsive, nanostructured phases driven by self-assembly would represent a significant advance in the understanding of this class of molecules, allowing for their expansion into applications such as controlled release and nanoscale templating.

Here, we report the high-concentration self-assembly behaviour of the AzoTAB, $\mathbf{C}_{8} \mathbf{A z o O C} \mathbf{C}_{2}$ TAB (Fig. 1a). This watersoluble AzoTAB has been synthesised previously and both the critical micelle concentrations $\left(\mathrm{CMC}_{\text {trans }}=0.3 \mathrm{mM} ; \mathrm{CMC}_{\text {cis }}=\right.$ $0.8 \mathrm{mM})^{26,33}$ and low-concentration self-assembly behaviour ${ }^{26}$ reported. It demonstrates the photochromic behaviour typical of azobenzenes (Fig. 1b), photoisomerising between the trans$\left(\lambda_{\max }=352 \mathrm{~nm}, \pi \rightarrow \pi^{*}\right)$ and cis-photostationary states $\left(\lambda_{\max }=\right.$ $316 \mathrm{~nm}, \pi \rightarrow \pi^{*} ; \lambda=435 \mathrm{~nm}, \mathrm{n} \rightarrow \pi^{*}$ ). After UV irradiation
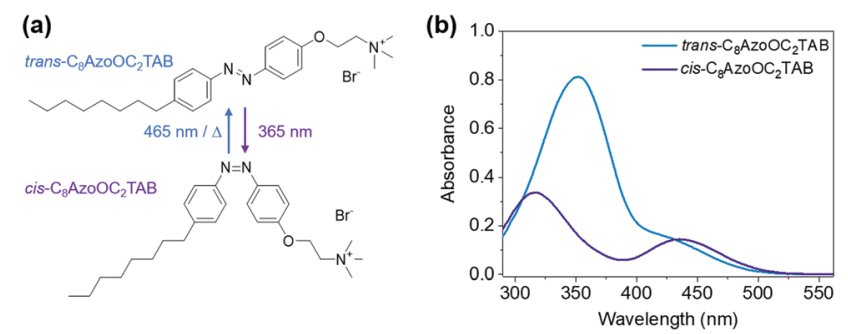

Fig. $1 \mathrm{C}_{8} \mathrm{AzoOC}_{2} \mathrm{TAB}$ photoisomerises between the cis- and trans-forms. (a) Molecular structure of trans- and cis- $\mathrm{C}_{8} \mathrm{AzoOC}_{2} \mathrm{TAB}$ and (b) UV-Vis absorption spectra of $\mathrm{C}_{8}$ AzoOC ${ }_{2}$ TAB $(30 \mathrm{mM})$ in the trans- (blue) and cis(purple) photostationary states. 
(365 $\mathrm{nm}, 1.5 \mathrm{~min}$ ) an 86\% cis-photostationary state (PSS, see $\mathrm{ESI} \dagger$ for calculation) is obtained and the system can recover a 94\% trans-PSS with blue light irradiation (465 nm, $3.0 \mathrm{~min}$ ). Full recovery from the cis-PSS to an assembly of $100 \%$ trans-isomers is possible by heating at $60{ }^{\circ} \mathrm{C}$ for one hour (Fig. S1, ESI $\dagger$ ). The firstorder rate constants of the forward and reverse reactions were determined to be $k_{\text {trans-cis }}=0.077 \mathrm{~s}^{-1}$ and $k_{\text {cis-trans }}=0.021 \mathrm{~s}^{-1}$ (Fig. S2, ESI $\dagger$ ), similar to values obtained for neutral AzoPS. ${ }^{30}$

High concentration solutions of $\mathbf{C}_{8} \mathbf{A z o O C}_{2} \mathbf{T A B}$ (10-40 wt $\%$ in water) were analysed using polarised optical microscopy (POM), small-angle X-ray scattering (SAXS) and differential scanning calorimetry (DSC) to determine the phase behaviour in this concentration regime. Fig. 2a-d shows POM images of $\mathbf{C}_{8} \mathbf{A z o O C} \mathbf{C}_{2} \mathbf{T A B}$ (native trans-state) in aqueous solutions at 10-40 wt\%. The POM images show bright colours and textures, which change colour when rotated $90^{\circ}$ (Fig. S3, ESI $\dagger$ ). These indicate the formation of optically anisotropic, ordered phases, such as LLC phases. These phases are typically assigned by the nature of the textures observed and by monitoring changes in the texture. ${ }^{28-30}$ At $10 \mathrm{wt} \%$, a smoke-like texture can be observed, which changes to a mosaic pattern at $20 \mathrm{wt} \%$. Both smoke-like and mosaic patterns are typical of a hexagonal LLC phase. ${ }^{34,35}$ At $30 \mathrm{wt} \%$, a classic conic fan-like texture is present, characteristic of a hexagonal phase. At $40 \mathrm{wt} \%$, the texture reverts to a smoke-like one, suggesting that a hexagonal phase is maintained for all concentrations probed here. Additional POM images for 10-40 wt\% are available in the ESI $\dagger$ (Fig. S3), showing the consistency of these textures for a given concentration. It is anticipated that when the close-packing limit of cylinders is reached at higher concentrations, and as the hydration and size of the head group decreases, a phase change from hexagonal to the lower curvature lamellar phase will be observed. $^{36}$

SAXS profiles for 10, 15 and $20 \mathrm{wt} \%$ solutions of $\mathbf{C}_{8} \mathbf{A z o O C}_{2} \mathbf{T A B}$ are shown in Fig. 2e. All three scattering profiles have a strong peak at lower values of the scattering vector, $q$, which shifts to higher $q$, from $0.35 \mathrm{~nm}^{-1}$ at $10 \mathrm{wt} \%$ to $0.45 \mathrm{~nm}^{-1}$ at $20 \mathrm{wt} \%$, with increasing concentration. This peak shift corresponds to decreasing physical distances, from $18 \mathrm{~nm}$ to $14 \mathrm{~nm}$, as $q=2 \pi / d$, where $d$ corresponds to real-space distance. This shift coincides with a concurrent decrease in the peak intensity and the growth of a small, yet sharp peak at $1.89 \mathrm{~nm}^{-1}(3.3 \mathrm{~nm})$, assigned as a Bragg peak. These features suggest the emergence of long-range order within the AzoTAB solution. The sharp peak at $1.89 \mathrm{~nm}^{-1}$ could also indicate residual crystallinity in the sample, however all samples appeared fully solubilised before measurement.

Typically, LLC mesophases are assigned from SAXS profiles using characteristic ratios of peak repetitions i.e. peaks occur at multiples of $1: 2: 3: 4$ for lamellar phases and $1: \sqrt{ } 3: 2: \sqrt{ } 7$ for hexagonal phases. ${ }^{37}$ In this case, there are insufficient peaks available to assign an LLC phase with certainty. However, some assumptions can be made to account for the absence of definitive peak spacing ratios in the SAXS profiles: (1) it is not uncommon for such systems to form swollen phases, ${ }^{38}$ and therefore have obscured scattering peaks; (2) it is expected that more Bragg peaks will emerge at higher concentrations (for neutral AzoPS multiple peaks develop in the SAXS profiles from $30-50 \mathrm{wt} \%$ onwards). ${ }^{28-30}$ This is a lower concentration compared to their non-photoactive analogues, alkyl-TAB surfactants, where the formation of LLC mesophases was only observed for concentrations above $50 \mathrm{wt} \%,{ }^{39}$ most likely due to the presence of the azobenzene group, which facilitates $\pi-\pi$ stacking. Unfortunately, above $20 \mathrm{wt} \%$ the samples were extremely viscous and could not be loaded into the SAXS instrument. However, this observation is indicative of interesting phase behaviour, as LLC mesophases often have high viscosities. ${ }^{28}$

The native trans-state solutions were then irradiated with UV light $(\lambda=365 \mathrm{~nm})$ to form the cis-PSS. Fig. 3a presents a series of POM images showing the step-by-step transformation

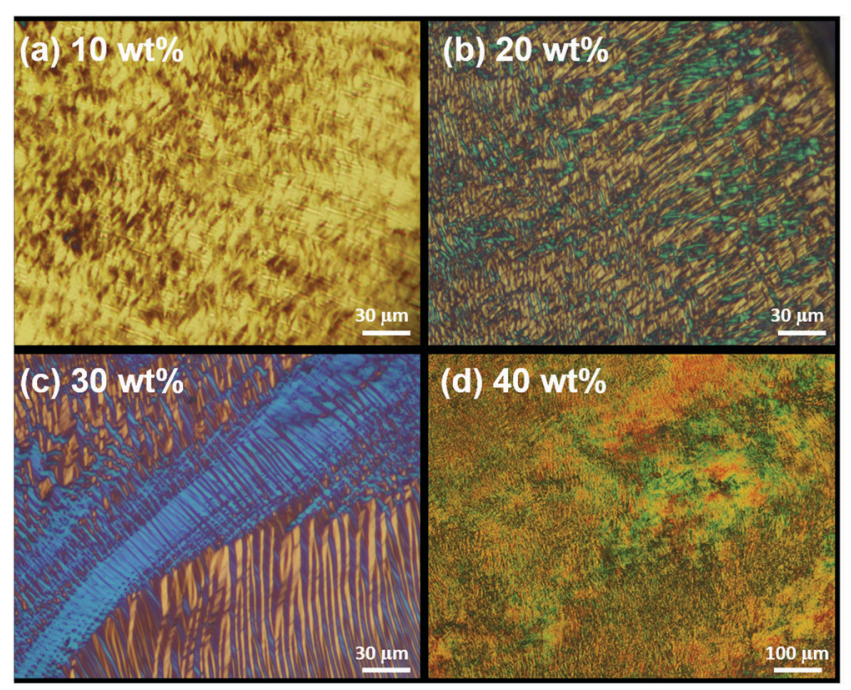

(e)

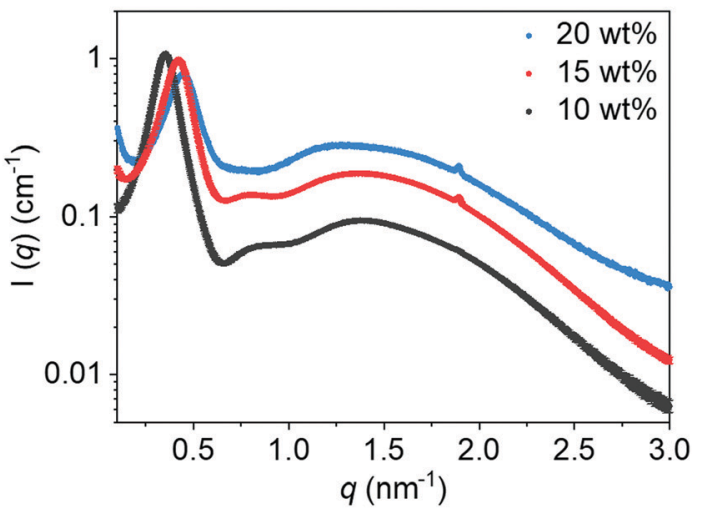

Fig. 2 POM images of trans $-\mathrm{C}_{8} \mathrm{AzOOO} \mathrm{C}_{2} \mathrm{TAB}$ in water at (a) $10 \mathrm{wt} \%$, (b) $20 \mathrm{wt} \%$, (c) $30 \mathrm{wt} \%$ and (d) 40 wt\% exhibiting optical anisotropy and a variety of textures. (e) Effect of concentration on the SAXS profiles of trans- $\mathbf{C}_{8} \mathbf{A z o O C}_{\mathbf{2}} \mathrm{TAB}$ in $\mathrm{H}_{2} \mathrm{O}$ at $10 \mathrm{wt} \%$ (grey), 15 wt\% (red) and 20 wt\% (blue) in water. Temperature $=20^{\circ} \mathrm{C}$. 
(a)

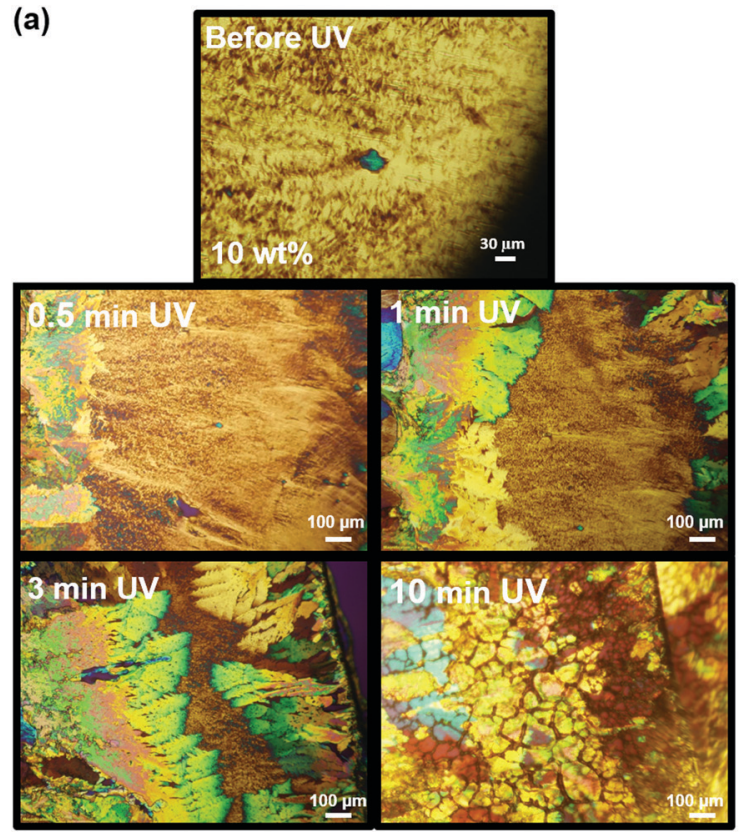

(b)

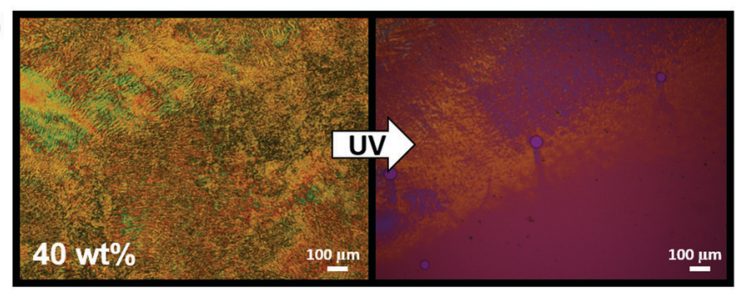

(c)

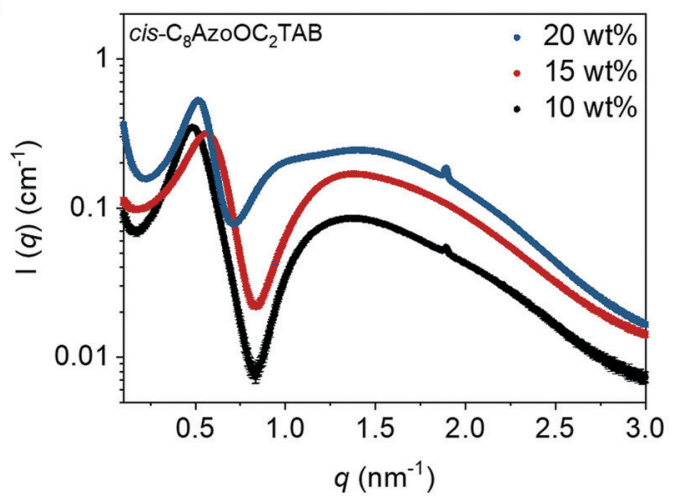

Fig. 3 Effect of UV irradiation on the self-assembly behaviour of $C_{8} A z O O C_{2} T A B$ in aqueous solution. (a) POM images of 10 wt\% $C_{8} A z o O C_{2} T A B$ after intervals of UV irradiation, (b) POM images before and after UV irradiation of $C_{8} A z O O C_{2} T A B\left(40\right.$ wt\%), (c) SAXS profiles of $C_{8} A z o O C_{2}$ TAB in the cis-PSS at $10 \mathrm{wt} \%$ (black), $15 \mathrm{wt} \%$ (red) and $20 \mathrm{wt} \%$ (blue).

of $\mathbf{C}_{8} \mathbf{A z o O C}_{2} \mathbf{T A B}$ at $10 \mathrm{wt} \%$ as a function of UV irradiation time. The smoke-like texture of the trans-isomer is present before irradiation. After $0.5 \mathrm{~min}$ irradiation, a strongly anisotropic region with bright colours emerges on the left-hand side of the image and the smoke-like pattern changes to a more streaked texture. After $1 \mathrm{~min}$ of UV irradiation, the brightly coloured, fan-like domain continues to grow and by $3 \mathrm{~min}$, it covers almost the entire image, with only a small region of the initial smoke-like texture remaining. The UV irradiation was continued for 10 minutes, resulting in a bright, dappled texture. This implies that an anisotropic phase remains for the cis-PSS, as a dark, untextured image would be expected if the LLC phases were completely disrupted. Fig. 3b shows the POM image for $\mathbf{C}_{8} \mathbf{A z o O C}_{2} \mathbf{T A B}$ at $40 \mathrm{wt} \%$ before and after UV irradiation. After UV irradiation a much darker image is formed, with a small amount of the underlying smoke-like texture remaining. In this case it seems that the initial LLC phase has been significantly disrupted upon photoisomerisation, with no indication of a new anisotropic LLC phase being formed. This variation in UV response highlights the different concentration-dependent self-assembly behaviour of the cis- and trans-isomers. The change in phase before and after UV irradiation is more significant at $40 \mathrm{wt} \%$ than at $10 \mathrm{wt} \%$.

The SAXS profiles of $\mathbf{C}_{8} \mathbf{A z o O C} \mathbf{C}_{2} \mathbf{T A B}$ at 10, 15 and $20 \mathrm{wt} \%$ after UV irradiation $(\lambda=365 \mathrm{~nm})$ are presented in Fig. $3 \mathrm{c}$. The first peak at low $q$ is shifted towards high $q$ compared to the trans-form, corresponding to smaller distances (13.9, 11.2 and $12.0 \mathrm{~nm}$ for 10,15 and $20 \mathrm{wt} \%$, respectively). A shift to self-assembled structures with smaller dimensions upon photoisomerisation has been observed for AzoTABs at lower concentrations $e . g$. decrease in micellar radius. ${ }^{26,27}$ The high $q$ diffraction peak emerges at the same value as for the trans-isomer $\left(1.89 \mathrm{~nm}^{-1}\right)$ but starts to appear at a lower concentration after UV irradiation. In this case, $15 \mathrm{wt} \%$ deviates from the trend of a shift of the initial peak to higher $q$ with increased concentration and is also the only profile to not have a Bragg peak at high $q$. A possible explanation is that a phase transition occurs at this concentration. The shift of the initial peak at $\sim 0.5 \mathrm{~nm}^{-1}$ back to low $q$ at $20 \mathrm{wt} \%$ tends to support this explanation.

For the $20 \mathrm{wt} \%$ sample, peak shoulders begin to emerge at 0.9 and $1.4 \mathrm{~nm}^{-1}$ in the SAXS profile. At this concentration, the peaks have a ratio in their positions of $1: 1.73: 2.6: 3.6$. This repetition corresponds with that of a hexagonal phase $(1: \sqrt{ } 3: 2: \sqrt{ } 7: \sqrt{ } 13$ ) except that the third peak (multiple of 2 ) is absent. The emergence of these shoulders at $20 \mathrm{wt} \%$ imply that, similar to the trans-form, at higher concentrations further Bragg peaks would develop in the SAXS profiles, supported by the high viscosities that made concentrations $>20 \mathrm{wt} \%$ impossible to load into the sample holder. It can be concluded that LLC phases are disrupted with UV light, either forming a different phase or being lost completely. Future work will focus on determining what factors are responsible for each scenario. It is likely that the susceptibility of the trans-form to disruption with UV light depends on the mesophase present e.g. lamellar or hexagonal. ${ }^{31}$

The stability of the LLC phases to temperature was also investigated. SAXS measurements were performed on the $20 \mathrm{wt} \%$ solution in the native trans-state at $10{ }^{\circ} \mathrm{C}$ intervals between 20 and $60{ }^{\circ} \mathrm{C}$. The lifetime of the cis-isomer was determined to be 1 hour at $60{ }^{\circ} \mathrm{C}$, before thermal relaxation to the more stable trans-form occurs. As the SAXS sample environment required relatively slow heating rates and equilibration times at each temperature, 
the effect of temperature was not studied after UV irradiation. It was found that for the $20 \mathrm{wt} \%$ sample, the peak at $q=1.89 \mathrm{~nm}^{-1}$ did not change position with increasing temperature, but rather a gradual decrease in intensity was observed for all scattering profiles (Fig. S4, ESI $\dagger$ ), suggesting looser packing, characteristic of a fluid state. ${ }^{40,41}$ DSC thermograms for $\mathbf{C}_{\mathbf{8}} \mathbf{A z o O C} \mathbf{C}_{\mathbf{2}} \mathbf{T A B}$ at 20 and $30 \mathrm{wt} \%$ show no peaks in the range of $10-80{ }^{\circ} \mathrm{C}$, indicating no melting or phase transition had occurred, in agreement with the lack of a structural transition in the SAXS profiles (Fig. S4, ESI $\dagger$ ).

In conclusion, the high-concentration phase behaviour of a cationic AzoPS has been reported for the first time. $\mathbf{C}_{\mathbf{8}} \mathbf{A z o O C _ { 2 }} \mathbf{T A B}$ forms long-range, anisotropic structures at high concentrations, assigned as LLC phases, which are altered or lost after UV irradiation. Future work will focus on studying the SAXS behaviour of $\mathbf{C}_{\mathbf{8}} \mathbf{A z o O C} \mathbf{C}_{\mathbf{2}} \mathbf{T A B}$, and related AzoTABs, above $30 \mathrm{wt} \%$, as an alternative sample holder has been developed to overcome the limitations of the high solution viscosity. This will progress our understanding of the phase behaviour of cationic AzoPS, allowing for dynamic, light-responsive systems with well-defined morphologies and dimensions to be constructed. The ultimate goal is to further extend the applications of AzoTABs into areas such as controlled uptake and release, catalysis and templating.

We acknowledge Diamond Light Source for time on Beamline B21 under proposal SM13867. C. B. thanks Trinity College Dublin for a postgraduate studentship award. E. A. K. thanks the Winton Programme for the Physics of Sustainability and the EPSRC (EP/N509620/1) for financial support. This work was supported in part by an Isaac Newton Trust/University of Cambridge Early Career Support Scheme grant.

\section{Conflicts of interest}

There are no conflicts to declare.

\section{References}

1 T. Yan, F. Li, S. Qi, J. Tian, R. Tian, J. Hou, Q. Luo, Z. Dong, J. Xu and J. Liu, Chem. Commun., 2019, 56, 149-152.

2 J. Lee, K. H. Ku, J. Kim, Y. J. Lee, S. G. Jang and B. J. Kim, J. Am. Chem. Soc., 2019, 141, 15348-15355.

3 X. Xie, L. Wang, X. Liu, Z. Du, Y. Li, B. Li, L. Wu and W. Li, Chem. Commun., 2020, 56, 1867-1870.

4 (a) M. Villa, G. Bergamini, P. Ceroni and M. Baroncini, Chem. Commun., 2019, 55, 11860-11863; (b) T. Kunitake, Y. Okahata, M. Shimomura, A. Yasunami and K. Takarabe, J. Am. Chem. Soc., 1981, 103(18), 5401-5413.

5 L. Stricker, E. C. Fritz, M. Peterlechner, N. L. Doltsinis and B. J. Ravoo, J. Am. Chem. Soc., 2016, 138, 4547-4554.

6 F. G. Downs, D. J. Lunn, M. J. Booth, J. B. Sauer, W. J. Ramsay, R. G. Klemperer, C. J. Hawker and H. Bayley, Nat. Chem., 2020, 12, 363-371.

7 J. Baillet, A. Gaubert, D. M. Bassani, J. Verget, L. Latxague and P. Barthélémy, Chem. Commun., 2020, 56, 3397-3400.
8 B. K. Das, B. Pramanik, S. Chowdhuri, O. A. Scherman and D. Das, Chem. Commun., 2020, 56, 3393-3396.

9 F. Huang, W. C. Liao, Y. S. Sohn, R. Nechushtai, C. H. Lu and I. Willner, J. Am. Chem. Soc., 2016, 138, 8936-8945.

10 O. Rifaie-Graham, S. Ulrich, N. F. B. Galensowske, S. Balog, M. Chami, D. Rentsch, J. R. Hemmer, J. Read De Alaniz, L. F. Boesel and N. Bruns, J. Am. Chem. Soc., 2018, 140, 8027-8036.

11 E. Merino, Chem. Soc. Rev., 2011, 40, 3835-3853.

12 S. Yagai and A. Kitamura, Chem. Soc. Rev., 2008, 37, 1520-1529.

13 E. Tan, S. Amirjalayer, S. Smolarek, A. Vdovin, F. Zerbetto and W. J. Buma, Nat. Commun., 2015, 6, 5860.

14 I. K. Lednev, T.-Q. Ye, R. E. Hester and J. N. Moore, J. Phys. Chem., 1996, 100, 13338-13341.

15 G. S. Hartley, J. Chem. Soc., 1938, 633-642.

16 K. G. Yager and C. J. Barrett, J. Photochem. Photobiol., A, 2006, 182, 250-261.

17 M. Cárdenas, K. Schillen, T. Nylander, J. Jansson and B. Lindman, Phys. Chem. Chem. Phys., 2004, 6, 1603-1607.

18 Y. Zakrevskyy, J. Roxlau, G. Brezesinski, N. Lomadze and S. Santer, J. Chem. Phys., 2014, 140, 44906.

19 A. Diguet, N. K. Mani, M. Geoffroy, M. Sollogoub and D. Baigl, Chem. - Eur. J., 2010, 16, 11890-11896.

20 G. Pouliquen and C. Tribet, Macromolecules, 2006, 39, 373-383.

21 S. Rudiuk, H. Saito, T. Hara, T. Inoue, K. Yoshikawa and D. Baigl, Biomacromolecules, 2011, 12, 3945-3951.

22 A. Diguet, R. M. Guillermic, N. Magome, A. Saint-Jalmes, Y. Chen, K. Yoshikawa and D. Baigl, Angew. Chem., Int. Ed., 2009, 48, 9281-9284.

23 A. Venancio-Marques, F. Barbaud and D. Baigl, J. Am. Chem. Soc., 2013, 135, 3218-3223.

24 S. Chen, W. Zhang, C. Wang and S. Sun, Green Chem., 2016, 18, 3972-3980.

25 S. Chen, C. Wang, Y. Yin and K. Chen, RSC Adv., 2016, 6, 60138-60144.

26 C. Blayo, J. E. Houston, S. M. King and R. C. Evans, Langmuir, 2018, 34, 10123-10134.

27 C. T. Lee, K. A. Smith and T. Alan Hatton, Langmuir, 2009, 25, 13784-13794.

28 S. Peng, Q. Guo, T. C. Hughes and P. G. Hartley, Langmuir, 2014, 30, 866-872.

29 S. Peng, Q. Guo, P. G. Hartley and T. C. Hughes, J. Mater. Chem. C, 2014, 2, 8303-8312.

30 J. E. Houston, E. A. Kelly, M. Kruteva, K. Chrissopoulou, N. Cowieson and R. C. Evans, J. Mater. Chem. C, 2019, 7, 10945-10952.

31 L. W. Giles, J. B. Marlow, C. S. G. Butler, G. A. Turpin, L. De Campo, S. T. Mudie, C. F. J. Faul and R. F. Tabor, Phys. Chem. Chem. Phys., 2020, 22, 4086-4095.

32 T. Kato and J. M. J. Fréchet, J. Am. Chem. Soc., 1989, 111, 8533-8534.

33 T. Hayashita, T. Kurosawa, T. Miyata, K. Tanaka and M. Igawa, Colloid Polym. Sci., 1994, 272, 1611-1619.

34 T. Nylander and A. Khan, Langmuir, 2000, 10044-10054.

35 Y. Zhao, X. Yue, X. Wang and X. Chen, J. Colloid Interface Sci., 2013, 389, 199-205. 
36 D. J. Mitchell, G. J. T. Tiddy, L. Waring, T. Bostock and M. P. McDonald, J. Chem. Soc., Faraday Trans., 1983, 79, 975-1000.

37 S. T. Hyde, Handb. Appl. Surf. Colloid Chem., 2001, 299-332.

38 Y. Uchida, T. Nishizawa, T. Omiya, Y. Hirota and N. Nishiyama, J. Am. Chem. Soc., 2016, 138, 1103-1105.
39 D. Varade, K. Aramaki and C. Stubenrauch, Colloids Surf., A, 2008, 315, 205-209.

40 F. G. Wu, J. S. Yu, S. F. Sun and Z. W. Yu, Langmuir, 2011, 27, 14740-14747.

41 S. M. Sagnella, C. E. Conn, I. Krodkiewska and C. J. Drummond, Soft Matter, 2009, 5, 4823-4834. 